\title{
Gas molecule effects on field emission properties of single-walled carbon nanotube
}

\author{
Chun-Wei Chen ${ }^{\mathrm{a}, *}$, Ming-Hsien Lee ${ }^{\mathrm{b}}$, S.J. Clark \\ ${ }^{a}$ Department of Materials Science and Engineering, National Taiwan University, 1 Rooselvelt Road, Sec. 4, Taipei, Taiwan, ROC \\ ${ }^{\mathrm{b}}$ Department of Physics, Tamkang University, Tamsui, Taipei, Taiwan, ROC \\ ${ }^{\mathrm{c}}$ Department of Physics, University of Durham, Durham DH1 3LE, UK
}

\begin{abstract}
The effective workfunctions of single-walled carbon nanotubes $(5,5)$ (SWNTs) with various geometries and adsorbates under external electric field have been calculated by the ab initio plane-wave, pseudopotential method. The capped, open-ended, and close-ended nanotubes show the workfunctions of $4.8 \mathrm{eV}, 4.43 \mathrm{eV}$ and $3.75 \mathrm{eV}$, respectively, and these results exhibit a good agreement with experiments. Under external electric field, the effective workfunction is further reduced due to the charge redistribution at the nanotube tip. In addition, the effects of participation of foreign adsorbates on the nanotube surface both physically and chemically on the variations of workfunctions have also been studied. In the physisorption process, the electrostatic interaction between adsorbates and nanotubes plays an important role under external electric field. The polar molecules like water have a large binding energy with the nanotube under electric field. These molecules act as tunneling states for electrons emitting from the nanotube tip into the vacuum. In the chemisorption process, the variations of effective workfunctions can be understood in terms of the surface dipole of the terminated bond due to the different electronegativity between nanotubes and adsorbates.

(C) 2003 Elsevier B.V. All rights reserved.
\end{abstract}

Keywords: Carbon nanotube; Filed emission; Workfunction; First-principles

\section{Introduction}

Carbon nanotubes have attracted considerable attention due to their unique geometry and prominent electronic properties, which demonstrate the potential applications in field emission displays [1] and other vacuum microelectronic devices [2]. Its high aspect ratio leads to a large electric field enhancement and a low emission threshold voltage. The other important factor, which governs the field emission behavior, is the workfunction of the emitter. Field emission from carbon nanotubes has been described by the Folwer-Nordheim tunneling theory [3], suggesting that emission act as a traditional tunneling process through high-aspect ratio carbon nanomaterials, with a workfunction similar to that of graphite. However, a more complicated emission mechanism is required to account for the observed changes in the slope of current-voltage characteristics. It is believed that adsorbates on the surface may be

\footnotetext{
*Corresponding author. Tel.: + 886-2336-65205; fax: + 886-223634562 .

E-mail address: chunwei@ntu.edu.tw (C.-W. Chen).
}

responsible for this behavior. For example, $\mathrm{H}_{2}$ molecules do not affect the emission behavior noticeably but water vapor, however, is found to significantly enhance the emission current. Although the workfunction of a filed emitter can be derived directly from the current-voltage characteristics, the uncertainty of local geometry of nanotubes leads to the unreliability of obtaining workfunction directly from the Fowler-Nordheim model. Several groups have attempted to estimate the workfunctions of single-walled nanotubes (SWNTs) and multiwalled nanotubes (MWNTs) by more sophisticated experiments. The values of workfunctions obtained vary from 3.7 to $5.6 \mathrm{eV}[4,5]$. In this article, we have sought to apply the ab initio, plane-wave, density functional theory (DFT) program to investigate the variations of field emission properties of SWNTs due to the influence of foreign adsorbates in terms of chemisorption and physisorption.

\subsection{Theoretical approaches and simulation models}

The calculations are performed using the CASTEP code $[6,7]$, which is a plane-wave, pseudopotential 


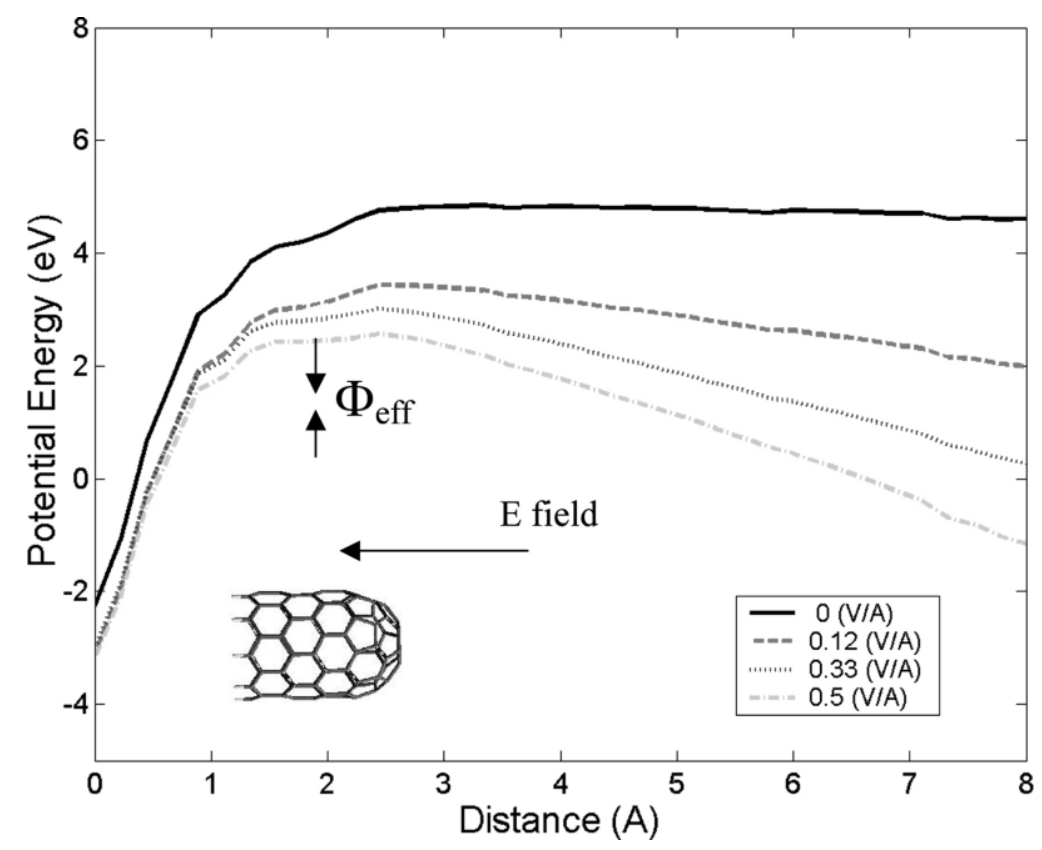

Fig. 1. Potential-energy curves for difference external electric field applied on a capped $(5,5)$ single-walled carbon nanotube tip. The Fermi energy is located at $0 \mathrm{eV}$. The effective workfunction is defined as the distance between the top of the potential profile and the Fermi energy.

program based on density functional theory (DFT). Generalized gradient approximation (GGA) [8] is used with the exchange-correlation potential by Wang and Perdew (PW91) [9]. The ion-electron interaction is modeled by the non-local real space [10], ultrasoft pseudopotential [11]. A plane-wave basis with a cut-off energy of $340 \mathrm{eV}$ and $\Gamma$ point approximation is used for our calculations. The workfunction $\Phi$ of a metal surface is defined as the energy needed to take an electron from the Fermi level $\mu$ to the vacuum level, by $\Phi=\varphi-\mu$, where $\varphi$ is the electrostatic potential caused by a surface dipole resulting from the spilling-out of electron density at the metal surface [12,13]. Different from the solids, the surface of one SWNT is modeled by its wall and tip due to its unique geometrical characteristics. The electronic structure of a SWNT surface is then represented by that of its tip and wall. Therefore, the difference in potential energy of one electron between the highest occupied molecular orbital (HOMO) and the vacuum level is defined as the corresponding 'effective workfunction' which is different from the conventional definition of workfunction. The vacuum level in our calculations is defined as the average value of potential energy distributions at the vacuum region during which the potential energy nearly comes to a constant value, as shown in Fig. 1. Under external electric field, the potential energy curves along the tube axis are also shown in Fig. 1. The 'effective workfunction' is then defined as the difference between the top of the potential energy curve and the HOMO state.
In our calculation, the model of armchair $(5,5)$ singlewalled carbon nanotube is represented by a six-layer (60 atoms) stem with its mouth capped by half of the $\mathrm{C}_{60}$ molecule. The dangling bonds at the other end are saturated by hydrogen atoms to avoid the boundary effect, giving a final structure consisting of total 100 atoms. The structure is then constructed within a tetragonal supercell with a lattice constant $30 \AA$ along the $z$ axis to represent the vacuum slab and the separation of $12 \AA$ along the $x$ - and $y$-axes to avoid the interaction between the two adjacent nanotubes. These atomic coordinates are fully optimized at zero electric field until the force on each atom during relaxation is less than $0.005 \mathrm{eV} / \AA$. The optimized geometry of a capped $(5,5)$ nanotube exhibits that the atoms at the top pentagon have an average bond length of $1.44 \AA$ compared to $1.42 \AA$ at the side wall. To study field emission properties of the carbon nanotube, an uniform external electric field is applied along with the tube axis by using a sawtooth-type potential to be compatible with the periodic condition. Structural changes are rather small even under the external electric field $\sim 0.75 \mathrm{~V} / \AA$. The local field strength around the tip is higher than the external applied field due to the small dimension of the tube tip. This effect is more enhanced with the increasing aspect ratio of carbon nanotubes. We calculated the maximum field strength outside the capped nanotube as a function of external electric field strengths. A near linear dependence can be seen until the critical external field strength $\sim 0.75 \mathrm{~V} / \mathrm{A}$ is achieved. The maximum field strength outside the capped end of the carbon 


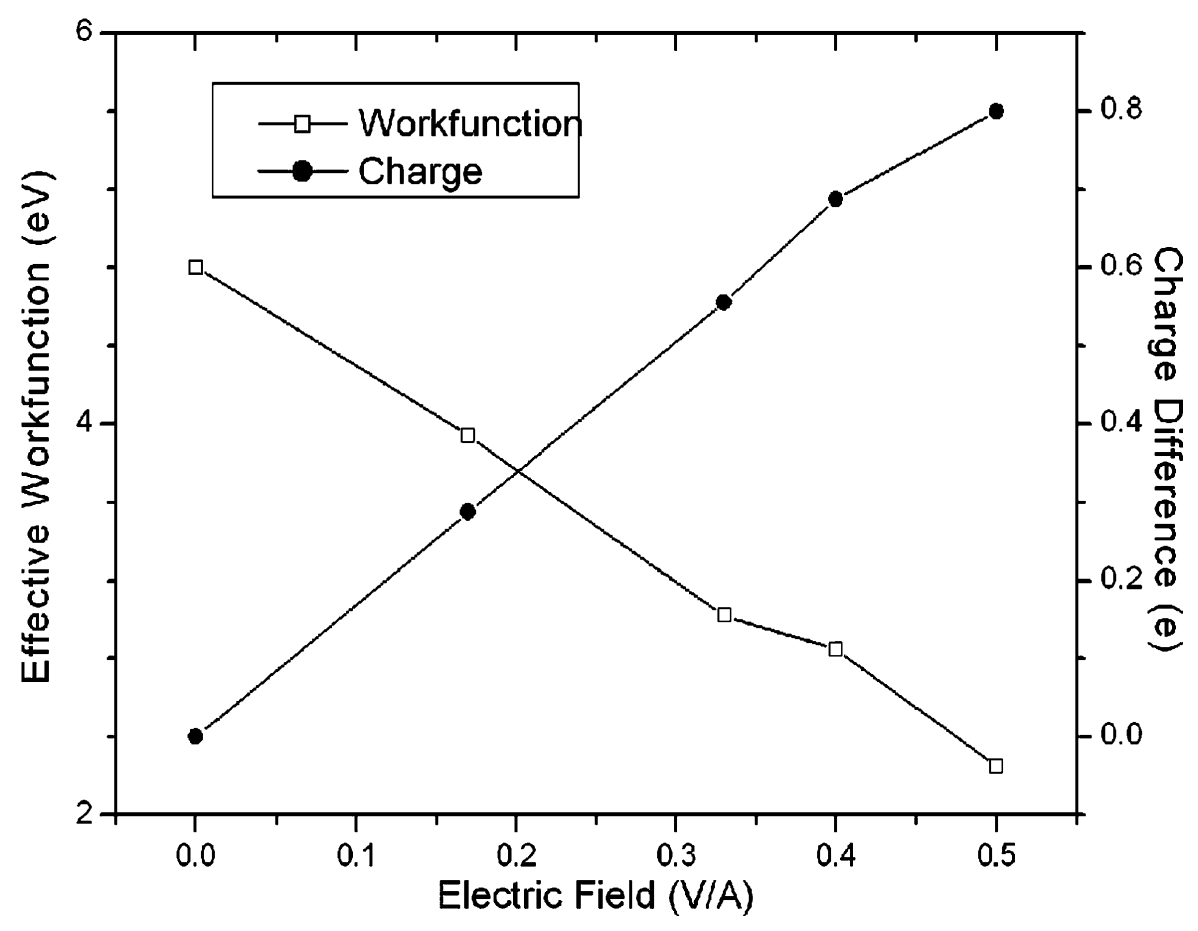

Fig. 2. Variations of the effective workfunction of a capped (5,5) single-walled carbon nanotube tip and the corresponding charge redistribution under difference electric field.

nanotube at this applied field is approximately $2.5 \mathrm{~V} / \mathrm{A}$ due to the field enhancement at the tip. Beyond this critical field, electrons become unbound and the accumulation of electrons at the end of supercell can be found. In this new regime, the present computational approach, which employs a bound basis state, is no longer suitable.

$\Delta Q($ charge difference $)=Q($ with external field $)$

$-Q$ (without external field).

In the presence of external electric field, the electrons along the tube axis are redistributed and this leads to the variation of the effective workfunction as well. Fig. 2 shows the charge redistribution along the capped nanotube axis under different applied electric fields and the magnitude of redistributed charge $\Delta Q$ is found nearly linear to the applied external field strengths. It can be seen that the nanotube tube is now polarized, with an increase charge density at the tip end and a decrease charge density at the bottom end. The polarization can be mainly attributed to charge redistribution of $\pi$ electrons along the tube axis. The increase of charge density at the tip end gives the states of higher occupied energy levels, resulting in the change of potential energy profile. The total potential energy curve in the system is mainly determined by two factors - the external potential and the electron-electron repulsion term induced by the accumulation of electrons at the tip. The degree of polarization along the tube axis is found to be stronglydependent on the applied field before the critical field is achieved. The effective workfunction defined as the difference between the Fermi level and the top of potential profile is then reduced with the applied field due to the accumulation of charge at the tube tip, which raises the Fermi level of CNTs. The capped CNT has a higher workfunction value compared to the open-ended ones under no applied field. With the external electric field, the capped CNT now shows a lower value of workfunction than its open-ended counterpart due to a larger amount of charge redistribution in the capped end of CNTs. The field penetration into the fullerene-like nanotube tip leading to further reduction of effective workfunction accounts for the ideal emission application of CNTs compared to the metallic tip regarded as a perfect conductor where no field penetration is observed. The effect of band bending induced by field penetration near the semiconductor surface has been discussed by Tsong [14]. CNTs can, therefore, be an excellent field emitter candidate with the combination of the metallic tube body and semiconducting tip end. The former is good for electron transport, and the latter is good for 
Table 1

The effective of workfunction and the amount of charge redistribution of CNTs with and without external electric field

\begin{tabular}{llll}
\hline & CAP- $(5,5)$ & Open- $(5,5)$ & Open $+\mathrm{H}-(5,5)$ \\
\hline$\Phi(\mathrm{eV})(F=0 \mathrm{~V} / \mathrm{A})$ & 4.78 & 4.47 & 3.75 \\
$\Phi(\mathrm{eV})(F=0.33 \mathrm{~V} / \mathrm{A})$ & 3.02 & 3.30 & 2.01 \\
$\Delta$ charge $(\mathrm{e})$ & 0.55 & 0.37 & 0.63
\end{tabular}

CAP- $(5,5)$ represents the capped CNT. Open- $(5,5)$ represents the open-ended CNT. Open $+\mathrm{H}-(5,5)$ represents the closed-ended CNT. The bond length of $\mathrm{C}=\mathrm{C}$ at the mouth of the open-ended CNT is $1.23 \AA$.

electron emission due to the band bending occurring in field penetration.

To study the influence of different adsorbates on the workfunctions of CNTs, a systematic study of interactions between various adsorbates and the capped nanotube surface is carried out. Both physisorption and chemisorption on the carbon nanotube surface are taken into account. In the physisorption process, the adsorbate molecules, $\mathrm{H}_{2} \mathrm{O}$ and $\mathrm{H}_{2}$ are initially located at a distance of $1.5 \AA$ above the nanotube tip and reach to a distance $\sim 3 \AA$ above the nanotube tip after relaxation. This weak interaction primarily results from the electrostatic force in nature between molecules and nanotubes. To mimic the chemisorption on the nanotube surface, the top surface of the nanotube is terminated with $\mathrm{H}$ and $\mathrm{O}$ atoms, respectively. The structural changes of carbon atoms terminated with these adsorbate atoms now become significant in accompany with bond formations and breakings.

\section{Result and discussion}

\subsection{Geometric effect}

The calculated workfunctions with and without external electric field are summarized in Table 1 . The capped armchair nanotube is found to have a workfunction 4.78 $\mathrm{eV}$, which shows a good agreement with the recent experimental measurements of SWNTs bundles with a value of $4.8 \mathrm{eV}$ by Suzuki et al. [4] using the ultraviolet photoemission spectroscopy. Although the $(5,5)$ armchair nanotube is metallic, the capped nanotube is found to be semiconducting with an energy gap of $1.4 \mathrm{eV}$ between the HOMO and LUMO (lowest unoccupied molecular orbital), resulting from the half of a $\mathrm{C}_{60}$ molecule. The corresponding local charge densities of the HOMO and LUMO are found mostly localized at the side of the tip, not at the top of the hemisphere at zero electric field. The LUMO states turn out to be located at the capped end resulting from charge accumulation at the capped end, when the electric field is applied along the tube axis. The detailed electronic structure and the corresponding localized states at carbon nanotube tips have also been investigated by Ref. $[15,16]$.

The relaxed structure of the open-ended nanotube as shown in Table 1 has a smaller diameter at its mouth with a shorter $\mathrm{C}-\mathrm{C}$ bond length of $1.23 \AA$ than $1.43 \AA$ before relaxation. The formation of triple bonds at the tube mouth is also seen experimentally in the morphology of multiwalled carbon nanotubes [17]. The charge redistribution after structural relaxation of the openended nanotube is shown in Fig. 3, which indicates a substantial decrease of spilling-out of electron density at the nanotube tip after structural relaxation, which reduces the surface dipole. The valence electrons at the mouth of the open-ended nanotube give the states of higher occupied energy levels and this leads to a reduction of its workfunction with a value of $4.39 \mathrm{eV}$ at zero electric field. When the external electric field is applied, the open-ended CNT shows a higher effective workfunction compared to the capped ended CNT due
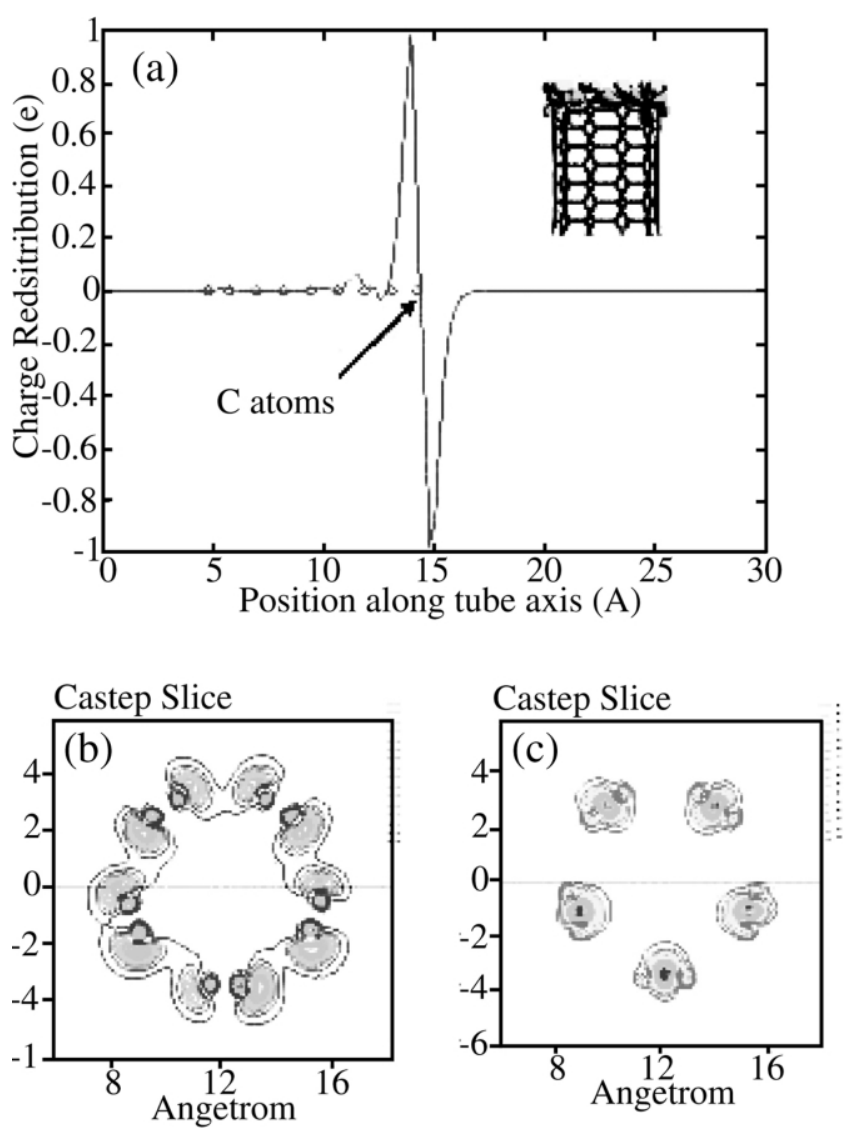

Fig. 3. Charge redistribution of an open-ended carbon nanotube after structural relaxation. (a) The increase of charge densities between neighboring $\mathrm{C}$ atoms indicates the formation of $\mathrm{C} \equiv \mathrm{C}$. The arrow indicates the average position of the top layer of relaxed carbon atoms. (b) shows the decrease of charge densities and (c) shows the increase of charge densities. 
to less amount of charge redistribution. The close-ended CNT exhibits a further reduction in its workfunction with a value of $3.75 \mathrm{eV}$ at zero electric field and the resulting lower workfunction can be attributed to the presence of a relatively small dipole on the $\mathrm{C}-\mathrm{H}$ bond, $\mathrm{C}^{\delta-}-\mathrm{H}^{\delta+}$, owing to the different electronegativity between $\mathrm{C}$ and $\mathrm{H}$. The potential difference across the dipole lower the distance between the Fermi energy and the vacuum level, leading to the reduction of workfunction. The effective workfunction of the close-ended CNT can be further reduced when the field along the axis is applied. Such enhanced field emission properties from CNT by hydrogen termination have also been reported by the recent experiment carried by Zhi et al. [18] using hydrogen plasma treatment on CNTs. Similar phenomena are also seen in the H-terminated diamond surface, which leads to its negative electron affinities [19]. Our result is opposite to the previous report by molecular orbital approximation, where the open-ended nanotube exhibits a lower workfunction than the close ended one [20]. This may attribute to that the tight-binding (TB) calculations in Ref. [20] are unlike to get the correct workfunction because TB does not use real orbitals and so does not give dipoles properly.

\subsection{Adsorbate effect}

We now discuss the variations of workfunctions of nanotubes owing to the participation of foreign molecules during field emission behavior. Current recovery experiments $[21,22]$ show that both physisorption and chemisorption are responsible for the observed currentvoltage curve deviation from the typical Folwer-Nordheim tunneling model. For example, the emission current is enhanced pronouncedly in the presence of water molecules, leading to a saturation of emission current. Such a current enhancement effect can be lowered after a higher temperature is applied, by removing the water adsorbates. This corresponds to the physisorption of water molecules on the nanotube surface. Hydrogen plasma treatment enhances field emission current has been reported by Zhi et al. [18] is believed to form $\mathrm{C}-\mathrm{H}$ bonds on the nanotube surface. In addition, a substantial current degradation after introducing oxygen gas results from the formation of $\mathrm{C}-\mathrm{O}$ dipoles on the nanotube surface. These correspond to chemisorption of hydrogen or oxygen atoms. In our study, both physisorption and chemisorption on the nanotube surface are taken into account.

Firstly, to explore the effect of physisorption on the carbon nanotube surface, water and $\mathrm{H}_{2}$ molecules are chosen to study their influence on the workfunction variations, respectively. The water molecule is initially located at a distance of $1.5 \AA$ above the cap and the fully relaxed models show that the water adsorbates migrate to a position of $\sim 3 \AA$ above the tube tip. The binding energy of an adsorbate is defined as:

$$
\begin{aligned}
E_{\text {binding }}\left(\mathrm{CNT}+\mathrm{H}_{2} \mathrm{O}\right)= & E_{\text {total }}\left(\mathrm{CNT}+\mathrm{H}_{2} \mathrm{O}\right)-E_{\text {total }}(\mathrm{CNT}) \\
& -E_{\text {total }}\left(\mathrm{H}_{2} \mathrm{O}\right)
\end{aligned}
$$

where $E_{\text {total }}\left(\mathrm{CNT}+\mathrm{H}_{2} \mathrm{O}\right)$ and $E_{\text {total }}(\mathrm{CNT})$ are the total energies of the carbon nanotube with and without a water adsorbate, respectively. $E_{\text {total }}\left(\mathrm{H}_{2} \mathrm{O}\right)$ is the total energy of an isolated water molecule. The binding energy of the water-nanotube system exhibits a value of $0.03 \mathrm{eV}$ at zero electric field, consistent with the experimental observation, in which the adsorption of water molecules on the CNT's surface is physisorption and it can be easily removed under a higher temperature. The workfunction of water-nanotube system at zero electric field shows a value of $4.91 \mathrm{eV}$ slightly higher than the clean nanotube of $4.78 \mathrm{eV}$. This is due to the polar nature of the water molecule with two positively charged hydrogen atoms 'pulling' the electron density out of the nanotube surface into the vacuum, raising its surface potential and workfunction.

The energy difference between the LUMO and the HOMO is approximately $1.4 \mathrm{eV}$ for both clean nanotube and water-nanotube systems at zero electric field. There is no significant enhancement of field emission properties in the water-nanotube system compared to the clean nanotube system at zero electric field. When we apply the external electric field at $0.33 \mathrm{~V} / \mathrm{A}$, the binding energy of water molecule with the nanotube significantly increases to a value of approximately $0.6 \mathrm{eV}$. The energy difference between the LUMO and the HOMO is now approximately $0.96 \mathrm{eV}$ compared to $1.16 \mathrm{eV}$ for the clean nanotube under the same electric field. The effective workfunction is approximately $2.6 \mathrm{eV}$. In addition to lowering the gap between the LUMO and the HOMO, the density of states (DOS) around the LUMO state in the water-nanotube system is also being enhanced significantly as shown in Fig. 4. These states resulting from water adsorbates are able to act as tunneling states for electrons emitting from the nanotube into the vacuum, which accounts for the observed field emission enhancement after introducing water adsorbates [23]. These results indicate that the observed field emission enhancement is mainly due to the strong interaction between polar water molecules and nanotubes under electric field. However, the non-polar $\mathrm{H}_{2}$ molecule, located parallel to the top pentagon surface, is found to have less influence on the workfunction of the nanotube at zero electric field, giving a value of $4.76 \mathrm{eV}$ similar to the clean nanotube of $4.78 \mathrm{eV}$. The binding energy does not show any significant variation as seen in the water-nanotube system after applying the same external field of $0.33 \mathrm{~V} / \mathrm{A} . \mathrm{H}_{2}$ molecules are known to physisorb on the outer surfaces of carbon nanotubes and are easily desorbed in a high field environment. This may explain 


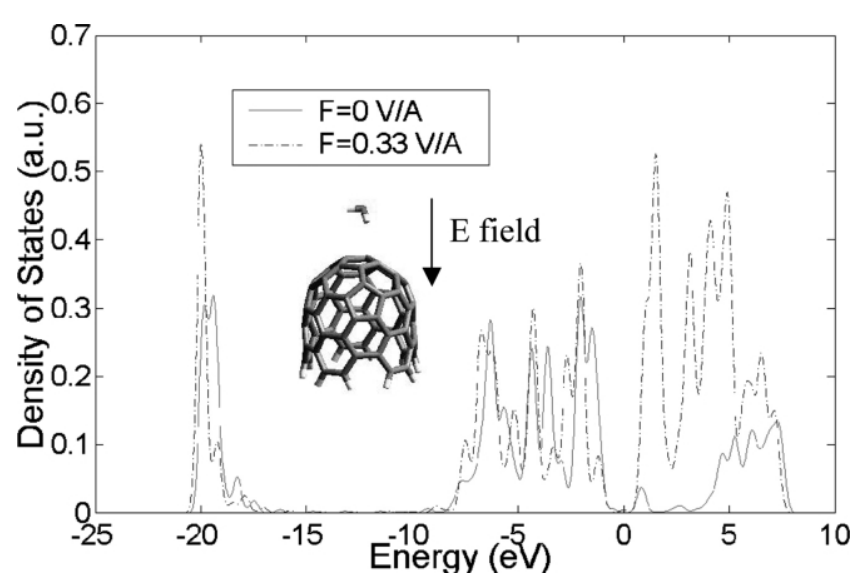

Fig. 4. The increase of density of states (DOS) of water molecule on the nanotube surface under external electric field of $0.33 \mathrm{~V} / \AA$. The Fermi energy is located at $0 \mathrm{eV}$. The density of states (DOS) of the water molecule with and without external electric field is approximately $2: 1$.

the lack of influence of $\mathrm{H}_{2}$ molecules on the field emission characteristics of carbon nanotubes. The variations of binding energy and energy gap of the waterand $\mathrm{H}_{2}$-nanotube systems are summarized in Table 2 .

We now consider the effect of chemisorption on the nanotube surface. The adsorbates under the high electric field during emission may result in several possible metastable forms, such as charged states or radicals. These metastable states may furthermore form chemical bonds with carbon atoms on the nanotube surface. The variations of workfunctions as a result of chemisorption between $\mathrm{C}$ atoms and different adsorbates are studied. The hydrogen-terminated capped structure has a $\mathrm{C}-\mathrm{H}$ bond length of $1.11 \AA$ and is found to have a lower workfunction of $3.95 \mathrm{eV}$ than $4.78 \mathrm{eV}$ for a clean capped nanotube. The $\mathrm{C}$ atoms vary from being a $\mathrm{sp}^{2}-$ like planar configuration before relaxation to being a $\mathrm{sp}^{3}$-like tetrahedral configuration after relaxation. The resulting lower workfunction can be attributed to the presence of a relatively small dipole on the $\mathrm{C}-\mathrm{H}$ bond, $\mathrm{C}^{\delta-}-\mathrm{H}^{\delta+}$, owing to the different electronegativity between $\mathrm{C}$ and $\mathrm{H}$ as mentioned above. The lower of workfunction will be more enhanced if more $\mathrm{C}-\mathrm{H}$ bonds are formed. The charge redistribution after forming $\mathrm{C}-\mathrm{H}$
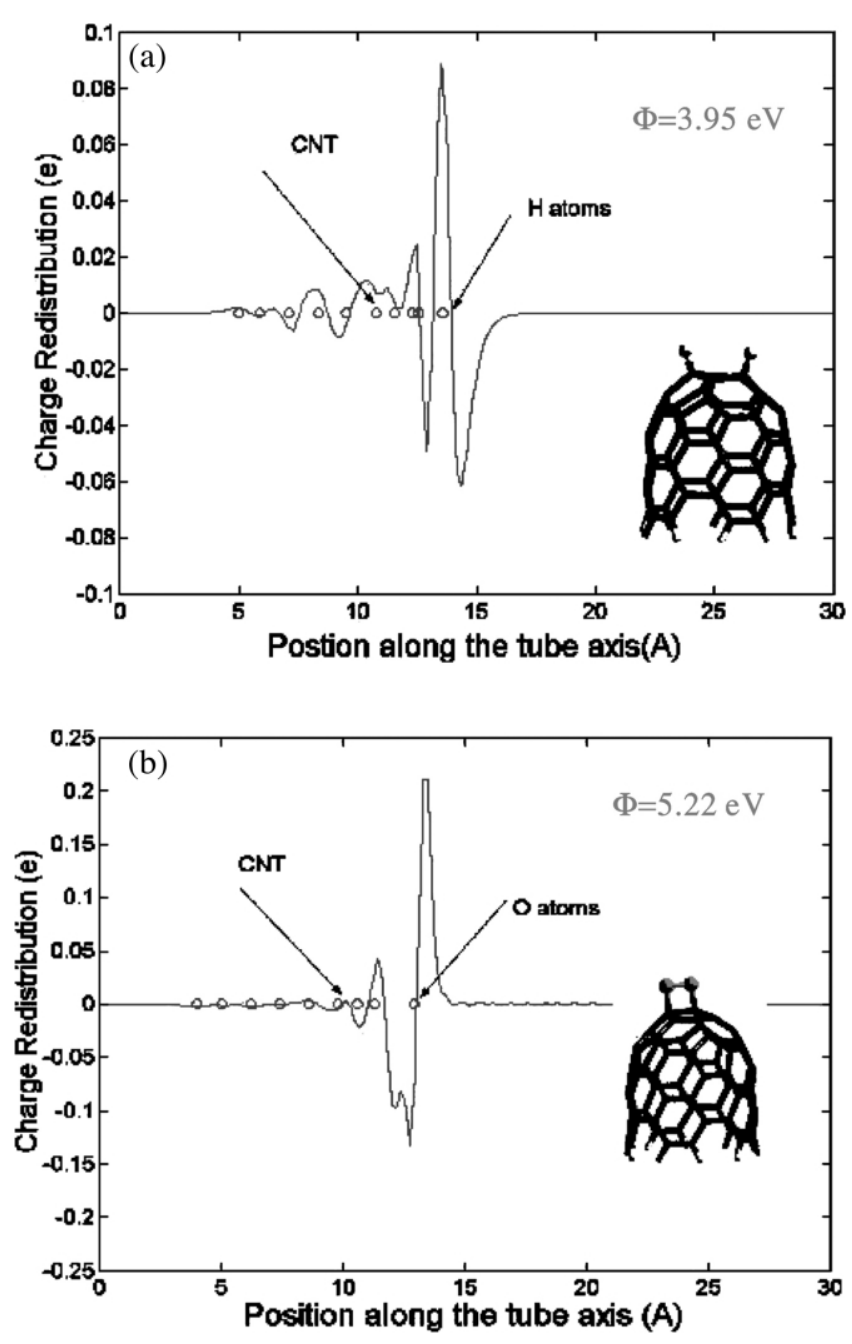

Fig. 5. The charge redistribution of the capped carbon nanotube surface terminated by hydrogen and oxygen atoms, respectively: (a) indicates that charge transfers from $\mathrm{H}$ atoms to $\mathrm{C}$ atoms. The circle at the position of $13.9 \AA$ along the axis indicates the average position of $\mathrm{H}$ atoms. The other circles indicate the average positions of $\mathrm{C}$ atoms along the axis; (b) shows that charge transfer from $\mathrm{C}$ atoms to $\mathrm{O}$ atoms. The circle at the position of $13.0 \AA$ along the axis indicates the average position of $\mathrm{O}$ atoms. The other circles indicate the average positions of $\mathrm{C}$ atoms along the axis.

\section{Table 2}

The binding energy and energy gap of clean CNT, CNT + water molecule and CNT + hydrogen molecule without and with electric field, respectively

\begin{tabular}{llll}
\hline & $\mathrm{CNT}($ clean$)(\mathrm{eV})$ & $\mathrm{CNT}+\mathrm{H}_{2} \mathrm{O}(\mathrm{eV})$ & $\mathrm{CNT}+\mathrm{H}_{2}(\mathrm{eV})$ \\
\hline$E_{\text {binding }}$ & & 0.03 & 0.01 \\
$E=0 \mathrm{~V} / \mathrm{A}$ & & 0.61 & 0.09 \\
$E=0.33 \mathrm{~V} / \mathrm{A}$ & & & 1.40 \\
$\Delta E_{\mathrm{g}}$ & 1.40 & 1.40 & 1.10 \\
$E=0 \mathrm{~V} / \mathrm{A}$ & 1.13 & 0.96 & \\
$E=0.33 \mathrm{~V} / \mathrm{A}$ & & & \\
\hline
\end{tabular}


bonds on the nanotube surface is shown in Fig. 5a. The charge is found to transfer from the hydrogen atoms to the carbon nanotube surface. The reduction of the workfunction is more significant under the external field of $0.33 \mathrm{~V} / \mathrm{A}$ with an effective workfunction of $2.25 \mathrm{eV}$. This result shows a good agreement with the experiment by hydrogen plasma treatment on carbon nanotubes, which enhances the field emission current [18]. It is found that the carbon nanotube is easily contaminated with oxygen atoms in the air by forming $\mathrm{C}-\mathrm{O}$ bonds on the surface. The model for the oxygen-terminated nanotube surface is shown in Fig. 5b. Oxygen molecules are now bonded with two carbon atoms on the tip to form a $\mathrm{C}-\mathrm{O}-\mathrm{O}-\mathrm{C}$ four-membered ring and the weaker $\pi$ bonds between two carbon atoms are replaced by two stronger $\mathrm{C}-\mathrm{O} \sigma$ bonds, exhibiting a four-fold configuration of carbon atoms. This model is found to have a workfunction $0.4 \mathrm{eV}$ higher than the clean nanotube. Due to the larger electronegativity of $\mathrm{O}$ atoms, the $\mathrm{C}-\mathrm{O}$ bond will produce a surface dipole, $\mathrm{C}^{\delta+}-\mathrm{O}^{\delta-}$, lowering the band energies inside the surface with respect to the fixed vacuum level. The corresponding charge redistribution after forming $\mathrm{C}-\mathrm{O}$ bonds on the nanotube surface is also shown in Fig. 5b. The charge is found to transfer from the carbon nanotube surface to oxygen atoms. The magnitude of increasing workfunction will become more pronounced if more oxygen molecules adsorb on the nanotube surface. This may account for experimental results that $\mathrm{O}_{2}$ exposure increases the turn-on filed of carbon nanotubes and lowers the emission current. Such current degradation results from the formation of $\mathrm{C}-\mathrm{O}$ dipoles, not from permanent structural damage. The above results indicate that $\mathrm{H}$-terminated surface will lower the workfunction of the carbon nanotube and Oterminated surface will raise the workfunction of the carbon nanotube.

\section{Conclusion}

In summary, we have performed the studies of workfunction variations of carbon nanotubes due to participation of foreign adsorbates including both physisorption and chemisorption based on ab initio, plane-wave pseudopotential, density functional theory calculations. The capped nanotube shows a workfunction of $4.78 \mathrm{eV}$ in a good agreement with experimental findings. The effective workfunction is lowered under external electric field due to charge redistribution at the surface. It is found that the nanotube tube is polarized under external electric field leading to charge redistribution. The band bending induced by field penetration into the nanotube tip surface can further reduce the effective workfunction of CNTs. The magnitude of redistributed charge $\Delta Q$ is found nearly linear to the applied external field strengths. In addition, the effects of adsorbates on the workfunctions of nanotubes exhibit different behavior between physi- sorption and chemisorption. In the physisorption process, the polar molecule like water interacts strongly with the carbon nanotube surface under electric field, which enhances field emission by increasing the density of states (DOS) around the Fermi level. These states are able to act as tunneling states for electrons emitting from the nanotube into the vacuum, which enhances field emission. For the non-polar $\mathrm{H}_{2}$ molecule, however, such a kind of enhancement cannot be achieved due to weak interaction. In the chemisorption process, the formations of chemical bonds between the adsorbate and nanotube lead to charge redistribution on the nanotube surface, by varying its surface dipole as well as its workfunction. It is found that $\mathrm{H}$-terminated surface will lower the workfunction and O-terminated surface will raise the workfunction, which may account for the observed substantial current enhancement after hydrogen plasma treatment and current degradation after oxygen exposure during field emission, respectively.

\section{Acknowledgments}

This work is supported by National Science Council, Taiwan with the project number of NSC90-2112-M155001.

\section{References}

[1] Q.H. Wang, A.A. Setlur, J.M. Lauerhaas, J.Y. Dai, E.W. Seelig, R.P.H. Chang, Appl. Phys. Lett. 72 (1998) 2912.

[2] Articles on nanotube in Phys. World 13 (2000) 29-53.

[3] R.H. Folwer, L. Nordheim, Proc. R. Soc. London, Ser. A 119 (1928) 683.

[4] S. Suzuki, C. Bower, Y. Watanabe, O. Zhou, Appl. Phys. Lett. 76 (2000) 4007

[5] M. Shiraishi, M. Ata, Carbon 39 (1913) 2001.

[6] M.C. Pyane, M. Teter, D.C. Allan, J.D. Joannopoulos, Rev. Mod. Phys. 64 (1992) 1045.

[7] V. Mailman, B. Winkler, J.A. White, C.J. Pickard, M.C. Payne, E.V. Akhmatskaya, et al., Int. J. Quant. Chem. 77 (2000) 895-910

[8] J.P. Perdew, J.A. Chevary, S.H. Vosko, K.A. Jackson, M.R. Pederson, D.J. Singh, et al., Phys. Rev. B46 (1992) 6671-6687.

[9] (a) Y. Wang, J.P. Perdew, Phys. Rev. B 43 (1991) 8911 (b) Y. Wang, J.P. Perdew, Phys. Rev. B 44 (1991) 13298.

[10] R.D. King-Smith, M.C. Pyane, J.S. Lin, Phys. Rev. B 44 (1991) 13063.

[11] D. Vanderbilt, Phys. Rev. B41 (1990) 7892-7895.

[12] N.D. Lang, Phys. Rev. B 4 (1971) 4234.

[13] J. Robertson, Diamond Relat. Mater. 5 (1996) 791.

[14] T.T. Tsong, Surf. Sci. 81 (1979) 28-42.

[15] C. Kim, B. Kim, S.M. Lee, C. Jo, Y.H. Lee, Phys. Rev. B 65 (2002) 165418

[16] D.L. Carroll, P. Redlich, P.M. Ajayan, J.C. Charlier, X. Blase, A. De Vita, R. Car, Phys. Rev. Lett. 78 (1997) 2811-2814.

[17] Y.-K. Kwon, Y.H. Lee, S.-G. Kim, P. Jund, D. Tománek, R.E. Smalley, Phys. Rev. Lett. 79 (1997) 2065-2068. 
[18] C.Y. Zhi, X.D. Bai, E.G. Wang, Appl. Phys. Lett. 81 (2002) 1690-1692.

[19] M.J. Rutter, J. Robertson, Phys. Rev. B 57 (1997) 9241.

[20] G. Zhou, W. Duan, B. Gu, Phys. Rev. Lett. 87 (2001) 095504.

[21] K.A. Dean, P. von Allmen, B.R. Chalmala, J. Vac. Sci. Technol. B 17 (1959) 1999.
[22] (a) K. Dean, B.R. Chalamala, Appl. Phys. Lett. 75 (1999) 3017

(b) K.A. Dean, B.R. Chalamala, J. Appl. Phys. 85 (1999) 3832.

[23] A. Maiti, J. Andzelm, N. Tanpipat, P. von Allmen, Phys. Rev. Lett. 87 (2001) 155502. 